\title{
Collisionless Dissipation of Radio-Frequency Waves in Axisymmetric Tokamak Plasmas
}

\author{
N.I. Grishanov ${ }^{1}$, C.A. de Azevedo ${ }^{2}$, and J.P. Neto ${ }^{1,2}$ \\ ${ }^{1}$ Laboratório Nacional de Computação Científica, 25651-070 Petrópolis, Brazil \\ ${ }^{2}$ Universidade do Estado do Rio de Janeiro, 20550-013 Rio de Janeiro, Brazil
}

Received on 26 June, 2001

\begin{abstract}
Contributions of trapped and untrapped particles to the transverse and longitudinal dielectric permittivity elements are present for radio-frequency waves in a tokamak with circular cross-sections of the magnetic surfaces and arbitrary aspect ratio. Imaginary parts of the longitudinal permittivity elements are important to estimate the wave power absorbed by electron Landau damping (e.g., during the plasma heating and current drive generation) in the frequency range of Alfvén, fast magnetosonic, and lower hybrid waves. Whereas, imaginary parts of the transverse permittivity elements are necessary to estimate the cyclotron-resonant wave dissipation at the fundamental cyclotron frequency of ions and/or electrons. The dissipated wave power is expressed by summation of terms including the separate contributions of trapped and untrapped particles to the imaginary parts of both the diagonal and non-diagonal elements of the dielectric permittivity. The concrete computations are carried out for a tokamak plasma with the main TCABR parameters.
\end{abstract}

\section{Introduction}

Tokamaks (both the large and small sizes) represent a promising alternative route to magnetic thermonuclear fusion. To achieve the fusion conditions in these devices an additional plasma heating must be employed. One of the effective heating schemes can be realized via the radio-frequency waves. To predict and analyze the power absorbed we should know the kinetic wave conductivity (or dielectric) tensor. For large aspect ratio tokamaks, solution of the Vlasov equation and dielectric tensor evaluation are developed quite completely (see, e.g., Refs. [1-4]) by using the smallness of the inverse aspect ratio: $a / R<<1$, where $a$ and $R$ are the minor and major radii of the plasma torus. In this paper, the transverse and longitudinal dielectric tensor elements are present for waves in a two-dimensional axisymmetric tokamak with circular magnetic surfaces in the case of arbitrary $a / R<1$. This model is especially suited the TCABR tokamak (having the circular plasma cross-sections) at the University of São Paulo, as well as other tokamaks with small elongation and triangularity.

\section{Plasma Model}

To describe an axisymmetric tokamak with circular magnetic surfaces we use the quasi-toroidal coordinates $(r, \theta, \phi)$, see Fig. 1, connected with the cartesian coordinates as

$$
\begin{gathered}
x=(R+r \cos \theta) \cos \phi, \\
y=(R+r \cos \theta) \sin \phi, \\
z=-r \sin \theta,
\end{gathered}
$$

where $r$ and $R$ are the minor and major radii of the magnetic surface, $\theta$ and $\phi$ are the poloidal and toroidal angles, respectively. The poloidal, $H_{0 \theta}$, and toroidal, $H_{0 \phi}$, projections of an equilibrium magnetic field $\mathbf{H}$, in this plasma model, are, respectively,

$$
\begin{aligned}
H_{0 \theta}(r, \theta) & =\frac{H_{\theta 0}(r)}{1+\epsilon \cos \theta}, \\
H_{0 \phi}(r, \theta) & =\frac{H_{\phi 0}(r)}{1+\epsilon \cos \theta} \\
\epsilon & =\frac{r}{R},
\end{aligned}
$$

satisfying the conditions: $\operatorname{div} \mathbf{H}=0$ and $(\operatorname{rot} \mathbf{H})_{r}=$ $\mathbf{n} \cdot \mathbf{H}=0$. In the $(r, \theta, \phi)$ coordinates, the modulus of an equilibrium magnetic field $\mathbf{H}$ is given as

$$
H(r, \theta)=\frac{\sqrt{H_{\phi 0}^{2}+H_{\theta 0}^{2}}}{1+\epsilon \cos \theta}
$$

where $H_{\phi 0}(r)$ and $H_{\theta 0}(r)$ correspond to the toroidal and poloidal magnetic field components at the points $\theta= \pm \pi / 2$ for a given (by $r$ ) magnetic surface. 

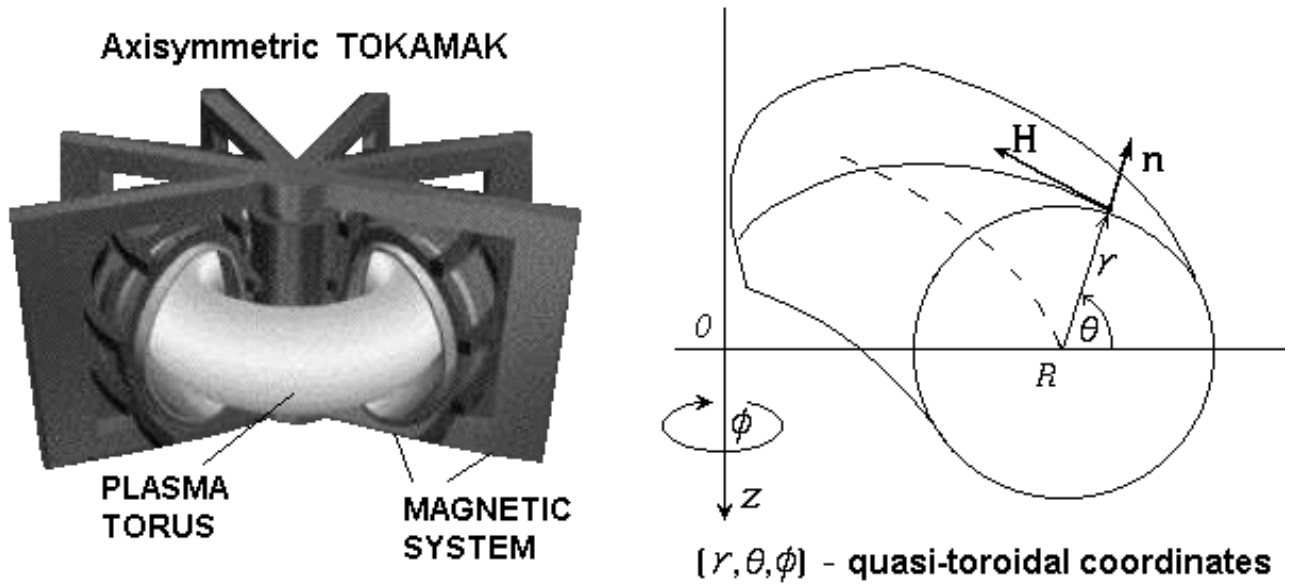

Figure 1. The quasi-toroidal coordinates $(r, \theta, \phi)$ for an axisymmetric tokamak plasma with circular magnetic surfaces. $\mathbf{n}$ is the unit vector normal to the toroidal magnetic surfaces $r=$ const.

Detailed evaluation of the transverse and longitudinal permittivity elements has been reported recently in Refs. [5, 6]. In contrast to Ref. [7] related to it, the Vlasov equation was solved separately for trapped and untrapped particles using i) the set of coordinates with the "straight" magnetic field lines, by introducing the new variable $\theta^{\prime}$ instead of $\theta$ as

$$
\theta\left(\theta^{\prime}\right)=2 \operatorname{arctg}\left[\sqrt{\frac{1+\epsilon}{1-\epsilon}} \operatorname{tg}\left(\frac{\theta^{\prime}}{2}\right)\right]
$$

and ii) new time-like variables $\tau_{u}\left(\theta^{\prime}\right)$ and $\tau_{t}\left(\theta^{\prime}\right)$ [the third kind elliptic integrals, Eqs. $(15,16)]$ for the untrapped and trapped particles, respectively, to describe the bounce periodic motion of each particle group along the magnetic field line. As a result of this procedure, the expressions of the dielectric tensor elements become simpler than those derived in Refs. [7, 8].

\section{Dielectric Tensor Elements}

To evaluate the dielectric characteristics we use the Fourier expansions of the perturbed current density and electric field in $\theta^{\prime}$ :

$$
\begin{aligned}
\frac{\mathbf{j}\left(r, \theta^{\prime}\right)}{1-\epsilon \cos \theta^{\prime}} & =\sum_{m}^{ \pm \infty} \mathbf{j}^{m}(r) \exp \left(i m \theta^{\prime}\right), \\
\frac{\mathbf{E}\left(r, \theta^{\prime}\right)}{1-\epsilon \cos \theta^{\prime}} & =\sum_{m^{\prime}}^{ \pm \infty} \mathbf{E}^{m^{\prime}}(r) \exp \left(i m^{\prime} \theta^{\prime}\right) .
\end{aligned}
$$

Note, the normal and binormal (to $\mathbf{H}$ ) current density components, $j_{n}$ and $j_{b}$ in our notation, are equal to $j_{n}=j_{1}+j_{-1}$ and $j_{b}=i\left(j_{1}-j_{-1}\right)$. The expressions for $\left.j_{l}\right|_{l= \pm 1}$ are convenient to analyze the cyclotron resonance effects at the fundamental cyclotron frequency of both the ions (if $l=-1$ ) and electrons (if $l=1$ ) in the explicit form. Accordingly, to estimate the $j_{l}$-components we use the left(right)-hand polarizated $E_{l}$-projections connected with $E_{n}$ and $E_{b}$ as $E_{l}=E_{n}-i l E_{b}$. As a result, the whole spectrum of $E$-field (by $\sum_{m^{\prime}}$ ) is present in a given (by $m$ ) harmonic of the current density:

$$
\begin{aligned}
& \frac{4 \pi i}{\omega} j_{l}^{m}(r)=\frac{4 \pi i}{\omega}\left[j_{l, u}^{m}(r)+j_{l, t}^{m}(r)\right]=\sum_{m^{\prime}}^{ \pm \infty}\left[\epsilon_{l, u}^{m, m^{\prime}}(r)+\epsilon_{l, t}^{m, m^{\prime}}(r)\right] E_{l}^{m^{\prime}}(r), \\
& \frac{4 \pi i}{\omega} j_{\|}^{m}(r)=\frac{4 \pi i}{\omega}\left[j_{\|, u}^{m}(r)+j_{\|, t}^{m}(r)\right]=\sum_{m^{\prime}}^{ \pm \infty}\left[\epsilon_{\|, u}^{m, m^{\prime}}(r)+\epsilon_{\|, t}^{m, m^{\prime}}(r)\right] E_{\|}^{m^{\prime}}(r) .
\end{aligned}
$$

Here $\epsilon_{l, u}^{m, m^{\prime}}, \epsilon_{l, t}^{m, m^{\prime}}$ and $\epsilon_{\|, u}^{m, m^{\prime}}, \epsilon_{\|, t}^{m, m^{\prime}}$ are the contributions of untrapped $(u)$ and trapped $(t)$ particles to the transverse and longitudinal dielectric permittivity elements, respectively:

$$
\epsilon_{l, u}^{m, m^{\prime}}=\frac{0.5 \omega_{L}^{2} r \sqrt{1+\epsilon}}{\pi^{2.5} \omega h_{\theta} v_{T} \sqrt{1-\epsilon}} \sum_{p}^{ \pm \infty} \int_{0}^{1} \frac{\kappa d \kappa}{\left(\kappa_{o}+\kappa\right)^{2}} \int_{-\infty}^{+\infty} \frac{u^{4} \exp \left(-u^{2}\right) A_{p, l}^{m} A_{p, l}^{m^{\prime}} d u}{\left(p+n q_{t}-l \delta-l q\right) u-U_{l}(\kappa)},
$$




$$
\begin{aligned}
\epsilon_{l, t}^{m, m^{\prime}} & =\frac{0.5 \omega_{L}^{2} r \sqrt{1+\epsilon}}{\pi^{2.5} \omega h_{\theta} v_{T} \sqrt{1-\epsilon}} \sum_{p}^{ \pm \infty} \int_{0}^{1} \frac{d \kappa}{\left(1+\kappa_{o} \kappa\right)^{2}} \int_{-\infty}^{+\infty} \frac{u^{4} \exp \left(-u^{2}\right)}{p u-V_{l}(\kappa)} B_{p, l}^{m} \hat{B}_{p, l}^{m^{\prime}} d u, \\
\epsilon_{\|, u}^{m, m^{\prime}} & =\frac{2 \omega_{L}^{2} r^{2} \sqrt{\kappa_{o}}(1+\epsilon)}{\pi^{3} h_{\theta}^{2} v_{T}^{2}(1-\epsilon)} \sum_{p}^{ \pm \infty} \int_{0}^{1} \frac{\Pi\left(\kappa_{o}, \frac{\pi}{2}, \kappa\right) C_{p}^{m} C_{p}^{m^{\prime}}}{\left(p+n q_{t}\right)^{2}\left(\kappa_{o}+\kappa\right)^{1.5}}\left[1+2 u_{p}^{2}+2 i \sqrt{\pi} u_{p}^{3} W\left(u_{p}\right)\right] d \kappa \\
\epsilon_{\|, t}^{m, m^{\prime}} & =\frac{4 \omega_{L}^{2} r^{2} \sqrt{\kappa_{o}}(1+\epsilon)}{\pi^{3} h_{\theta}^{2} v_{T}^{2}(1-\epsilon)} \sum_{p=1}^{\infty} \int_{0}^{1} \frac{\Pi\left(\kappa_{o} \kappa, \frac{\pi}{2}, \kappa\right) D_{p}^{m} D_{p}^{m^{\prime}}}{p^{2}\left(1+\kappa_{o} \kappa\right)^{1.5}}\left[1+2 v_{p}^{2}+2 i \sqrt{\pi} v_{p}^{3} W\left(v_{p}\right)\right] d \kappa .
\end{aligned}
$$

Here we have used the following definitions

$$
\begin{aligned}
& A_{p, l}^{m}(u, \kappa)=\int_{0}^{\pi} \cos \left\{\left(m+n q_{t}-l \delta\right) \eta-l g \theta(\eta)-\pi\left(p+n q_{t}-l \delta-l g\right) \frac{\tau_{u}(\kappa, \eta)}{\tau_{u}(\kappa, \pi)}-\right. \\
& \left.-l \frac{\Omega_{c} r \sqrt{2\left(\kappa_{o}+\kappa\right)}}{h_{\theta} u v_{T} \sqrt{\epsilon(1+\epsilon)}}\left[F\left(\frac{\eta}{2}, \kappa\right)-K(\kappa) \frac{\tau_{u}(\kappa, \eta)}{\tau_{u}(\kappa, \pi)}\right]\right\} \sqrt{\frac{1+\kappa_{o} \sin ^{2} \frac{\eta}{2}}{1-\kappa \sin ^{2} \frac{\eta}{2}}} d \eta, \\
& \hat{B}_{p, l}^{m}(u, \kappa)=\int_{0}^{\theta_{t}} \cos \left\{\left(m+n q_{t}-l \delta\right) \eta-l g \theta(\eta)-p \frac{\pi \tau_{t}(\kappa, \eta)}{2 \tau_{t}\left(\kappa, \theta_{t}\right)}-l \frac{\Omega_{c} r \sqrt{2\left(1+\kappa_{o} \kappa\right)}}{h_{\theta} u v_{T} \sqrt{\epsilon(1+\epsilon)}} \times\right. \\
& \left.\times\left[F\left(\arcsin \left(\sqrt{\frac{1}{\kappa}} \sin \frac{\eta}{2}\right), \kappa\right)-K(\kappa) \frac{\tau_{t}(\kappa, \eta)}{\tau_{t}\left(\kappa, \theta_{t}\right)}\right]\right\} \sqrt{\frac{1+\kappa_{o} \sin ^{2} \frac{\eta}{2}}{\kappa-\sin ^{2} \frac{\eta}{2}}} d \eta, \\
& B_{p, l}^{m}(u, \kappa)=\hat{B}_{p, l}^{m}(u, \kappa)+(-1)^{p} \hat{B}_{-p, l}^{m}(-u, \kappa), \quad W(z)=e^{-z^{2}}\left(1+\frac{2 i}{\sqrt{\pi}} \int_{0}^{z} e^{t^{2}} d t\right) \\
& \tau_{u}(\kappa, \eta)=\int_{0}^{\frac{\eta}{2}} \frac{d \alpha}{\left(1+\kappa_{o} \sin ^{2} \alpha\right) \sqrt{1-\kappa \sin ^{2} \alpha}}=\Pi\left(\kappa_{o}, \frac{\eta}{2}, \kappa\right), \quad \kappa_{o}=\frac{2 \epsilon}{1-\epsilon}, \\
& \tau_{t}(\kappa, \eta)=\Pi\left(\kappa_{o} \kappa, \arcsin \left(\frac{1}{\sqrt{\kappa}} \sin \frac{\eta}{2}\right), \kappa\right), \quad \theta_{t}(\kappa)=2 \arcsin \sqrt{\kappa}, \\
& C_{p}^{m}(\kappa)=\int_{0}^{\pi} \cos \left[\left(m+n q_{t}\right) \eta-\pi\left(p+n q_{t}\right) \frac{\tau_{u}(\kappa, \eta)}{\tau_{u}(\kappa, \pi)}\right] d \eta, \quad \Omega_{c}=\frac{e \sqrt{H_{\theta 0}^{2}+H_{\phi 0}^{2}}}{M c}, \\
& D_{p}^{m}(\kappa)=\int_{0}^{\theta_{t}} \cos \left[\left(m+n q_{t}\right) \eta-p \frac{\pi \tau_{t}(\kappa, \eta)}{2 \tau_{t}\left(\kappa, \theta_{t}\right)}\right] d \eta+ \\
& +(-1)^{p-1} \int_{0}^{\theta_{t}} \cos \left[\left(m+n q_{t}\right) \eta+p \frac{\pi \tau_{t}(\kappa, \eta)}{2 \tau_{t}\left(\kappa, \theta_{t}\right)}\right] d \eta \\
& U_{l}(\kappa)=\frac{r \sqrt{2\left(\kappa_{o}+\kappa\right)}}{\pi h_{\theta} v_{T} \sqrt{\epsilon(1+\epsilon)}}\left[\omega(1+\epsilon) \tau_{u}(\kappa, \pi)+l \Omega_{c} K(\kappa)\right], \quad h_{\theta}=\frac{H_{\theta 0}}{\sqrt{H_{\theta 0}^{2}+H_{\phi 0}^{2}}}, \\
& V_{l}(\kappa)=\frac{2 r \sqrt{2\left(1+\kappa_{o} \kappa\right)}}{\pi h_{\theta} v_{T} \sqrt{\epsilon(1+\epsilon)}}\left[\omega(1+\epsilon) \tau_{t}\left(\kappa, \theta_{t}\right)+l \Omega_{c} K(\kappa)\right], \quad h_{\phi}=\frac{H_{\phi 0}}{\sqrt{H_{\theta 0}^{2}+H_{\phi 0}^{2}}}, \\
& u_{p}(\kappa)=\frac{\omega r \sqrt{2(1+\epsilon)\left(\kappa_{o}+\kappa\right)}}{\left|p+n q_{t}\right| \pi h_{\theta} v_{T} \sqrt{\epsilon}} \tau_{u}(\kappa, \pi), \quad F(\eta, \kappa)=\int_{0}^{\eta} \frac{d \theta}{\sqrt{1-\kappa \sin ^{2} \theta}}, \\
& v_{p}(\kappa)=\frac{2 \omega r \sqrt{2(1+\epsilon)\left(1+\kappa_{o} \kappa\right)}}{p \pi h_{\theta} v_{T} \sqrt{\epsilon}} \tau_{t}\left(\kappa, \theta_{t}\right), \quad K(\kappa)=F\left(\frac{\pi}{2}, \kappa\right), \quad v_{T}=\sqrt{\frac{2 T}{M}}, \\
& \omega_{L}^{2}=\frac{4 \pi N e^{2}}{M}, \quad q_{t}=\frac{\epsilon h_{\phi} h_{\theta}^{-1}}{\sqrt{1-\epsilon^{2}}}, \quad \delta=\frac{1.5 h_{\phi}}{\sqrt{1-\epsilon^{2}}}, \quad g=\frac{h_{\phi}}{2}\left(\frac{1}{1-\epsilon^{2}}-\frac{r}{q_{t}} \frac{d q_{t}}{d r}\right) \text {. }
\end{aligned}
$$


Note, $\epsilon_{l, u}^{m, m^{\prime}}, \epsilon_{l, t}^{m, m^{\prime}}, \epsilon_{\|, u}^{m, m^{\prime}}, \epsilon_{\|, t}^{m, m^{\prime}}$ describe the contribution of any kind of untrapped and trapped particles to the dielectric tensor elements. The corresponding expressions for plasma electrons and ions can be obtained by replacing $T$ (temperature), $N$ (density), $M$ (mass), $e$ (charge) by the electron $T_{e}, N_{e}, m_{e}, e_{e}$ and ion $T_{i}, N_{i}, M_{i}, e_{i}$ parameters, respectively. To obtain the total expressions of the permittivity elements, as usual, it is necessary to carry out the summation over all species of plasma particles.

Since the phase coefficients $C_{p}^{m}(\kappa)$ and $D_{p}^{m}(\kappa)$, for the longitudinal permittivity elements, are independent of the wave frequency, $\omega$, and the particle energy, $v$, it is possible the analytical Landau integration of the perturbed distribution functions of both the trapped and untrapped particles in velocity space. As a result of this procedure, in contrast to Ref. [7] where the corresponding phase coefficients depend on $v, \epsilon_{\|, u}^{m, m^{\prime}}$ and $\epsilon_{\|, t}^{m, m^{\prime}}$ are written by the summation of bounce-resonant terms including the well known plasma dispersion function $W(z)$, i.e, by the probability integral of the complex argument, Eq. (14). After this, the numerical estimations of both the real and imaginary parts of the longitudinal permittivity elements become simpler, and their dependence on the wave frequency $\omega$ is defined only by the arguments $u_{p}(\kappa, \omega, \ldots)$ and $v_{p}(\hat{\kappa}, \omega, \ldots)$ of the plasma dispersion functions, $W\left(u_{p}\right)$ and $W\left(v_{p}\right)$. Introduction of the $W(z)$ function, in the standard integral form Eq. (14), became possible since the final equations for $f_{0, u}^{s}$ and $f_{0, t}^{s}$ (see Ref. [6]) have been reduced to the first order differential equations with respect to one variable $\tau_{u}$ or $\tau_{t}$, respectively, with the constant coefficients in the left-hand side. Another form of the coefficients $C_{p}^{m}(\kappa)$ and $D_{p}^{m}(\kappa)$ has been done in Appendix by the Jacobi elliptic functions, which also can be convenient in the computer calculations.

The bounce resonance conditions of the effective wave-particle interactions in a tokamak plamsa, as follows from Eqs. (4) and (5), are the same those derived in Ref. [8], and can be rewritten as

$$
\begin{gathered}
\left(p+n q_{t}-l \delta-l g\right) u-U_{l}(\kappa)=0, \\
l, p=0, \pm 1, \pm 2, \ldots
\end{gathered}
$$

for untrapped particles, where $u=v / v_{T}$, and

$$
p u-V_{l}(\hat{\kappa})=0, \quad l, p=0, \pm 1, \pm 2, \ldots
$$

for the trapped particles.

Since the whole spectrum of the E-field (by $\sum_{m^{\prime}}^{ \pm \infty}$ ) is present in a given (by $m$ ) current density harmonic, see Eqs. (6) and (7), it is necessary to take into account that the poloidal eigenmode numbers excited in a tokamak plasma may differ from the basic mode number(s) generated by the antenna system. Moreover, the excitation/dissipation of $E_{\|}^{\left(m^{\prime}\right)}$-harmonics with $m^{\prime} \neq m$ can lead to the additional heating of both the trapped and untrapped particles and destabilize a wide class of the low-frequency drift-Alfvén eigenmodes near the so-called rational magnetic surfaces, where the longitudinal wave vector component changes its sign, i.e., where $m+n q_{t}(r)=0$. This means that one-mode approximation (over $\theta$ ) is not valid to solve the Maxwell's equations in the tokamaks, in the general case. Nevertheless, there is some interest to compare the dielectric characteristics of a toroidal plasma (obtained by a two-dimensional consideration of the problem) and the corresponding characteristics of a cylindrical plasma model (one-dimensional consideration), which is well developed and often used for the study of the radiofrequency plasma heating and current drive problems in tokamaks in the frequency range of Alfvén, lower hybrid and ion-cyclotron waves.

\section{Wave Dissipation}

One of the main mechanisms of the radio-frequency plasma heating is the electron Landau damping of waves due to the Cherenkov resonance interaction of $E_{\|}$ with untrapped and trapped electrons. The Cherenkov resonance conditions are different for trapped and untrapped particles in tokamak geometry and have nothing general with the wave-particle resonance condition, $\omega=\left(h_{\theta} / r\right)\left(m+n q_{t}\right) v_{\|}$, in the cylindrical magnetized plasmas. As a result, after averaging in $t$ and $\theta^{\prime}$ and accounting for Eq. (7), the wave power dissipated by the electron Landau damping, $P_{\|}=\operatorname{Re}\left(E_{\|} j_{\|}^{*}\right)$, can be estimated as

$$
P_{\|}=P_{\|, u}+P_{\|, t}=\frac{\omega}{8 \pi} \sum_{m}^{ \pm \infty} \sum_{m^{\prime}}^{ \pm \infty}\left(\operatorname{Im} \epsilon_{\|, u}^{m, m^{\prime}}+\operatorname{Im} \epsilon_{\|, t}^{m, m^{\prime}}\right)\left[\operatorname{Re} E_{\|}^{m} \operatorname{Re} E_{\|}^{m^{\prime}}+\operatorname{Im} E_{\|}^{m} \operatorname{Im} E_{\|}^{m^{\prime}}\right]
$$

where, as follows from Eqs. (10) and (11),

$$
\operatorname{Im} \epsilon_{\|, u}^{m, m^{\prime}}=\frac{4 \omega_{L}^{2} r^{2} \sqrt{\kappa_{o}}(1+\epsilon)}{\pi^{2.5} h_{\theta}^{2} v_{T}^{2}(1-\epsilon)} \sum_{p}^{ \pm \infty} \frac{1}{\left(p+n q_{t}\right)^{2}} \int_{0}^{1} \frac{\Pi\left(\kappa_{o}, \frac{\pi}{2}, \kappa\right)}{\left(\kappa_{o}+\kappa\right)^{1.5}} u_{p}^{3} \exp \left(-u_{p}^{2}\right) C_{p}^{m} C_{p}^{m^{\prime}} d \kappa,
$$




$$
\operatorname{Im} \epsilon_{\|, t}^{m, m^{\prime}}=\frac{8 \omega_{L}^{2} r^{2} \sqrt{\kappa_{o}}(1+\epsilon)}{\pi^{2.5} h_{\theta}^{2} v_{T}^{2}(1-\epsilon)} \sum_{p=1}^{\infty} \frac{1}{p^{2}} \int_{0}^{1} \frac{\Pi\left(\kappa_{o} \kappa, \frac{\pi}{2}, \kappa\right)}{\left(1+\kappa_{o} \kappa\right)^{1.5}} v_{p}^{3} \exp \left(-v_{p}^{2}\right) D_{p}^{m}(\kappa) D_{p}^{m^{\prime}}(\kappa) d \kappa
$$

are the contribution of untrapped, $\operatorname{Im} \epsilon_{\|, u}^{m, m^{\prime}}$, and trapped, $\operatorname{Im} \epsilon_{\|, t}^{m, m^{\prime}}$, particles to the imaginary part of the longitudinal permittivity elements: $\operatorname{Im} \epsilon_{\|}^{m, m^{\prime}}=$ $\operatorname{Im} \epsilon_{\|, u}^{m, m^{\prime}}+\operatorname{Im} \epsilon_{\|, t}^{m, m^{\prime}}$. Thus, for the given $\omega, m, n, r$ and $E$-field amplitudes, the parts $P_{\|, u}$ and $P_{\|, t}$ differ by the different contributions of untrapped and trapped electrons to $\operatorname{Im} \epsilon_{\|}^{m, m^{\prime}}$ elements.

There is another important plasma heating mechanism due to the cyclotron wave damping in the range of ion/electron cyclotron frequencies, when the plasma particles interact effectively with the transverse electric field components, $E_{l}$. Under the cyclotron resonance heating (on the fundamental harmonics) the power absorbed, $P_{l}=\operatorname{Re}\left(E_{l} j_{l}^{*}\right)$, can be expressed as

$$
P_{l}=P_{l, u}+P_{l, t}=\frac{\omega}{8 \pi} \sum_{m}^{ \pm \infty} \sum_{m^{\prime}}^{ \pm \infty}\left(\operatorname{Im} \epsilon_{l, u}^{m, m^{\prime}}+\operatorname{Im} \epsilon_{l, t}^{m, m^{\prime}}\right)\left[\operatorname{Re} E_{l}^{m} \operatorname{Re} E_{\|}^{m^{\prime}}+\operatorname{Im} E_{l}^{m} \operatorname{Im} E_{l}^{m^{\prime}}\right]
$$

As was noted above, $l=-1$ corresponds to the wave power absorbed under the ion-cyclotron resonance heating when $\omega \sim \Omega_{c, i}$; whereas $l=1$ should be used under the electron-cyclotron plasma heating when $\omega \sim\left|\Omega_{c, e}\right|$. The contributions of untrapped and trapped particles to the imaginary parts of the transverse permittivity can be readily derived from Eqs. (8) and (9) by using the well known residue (or Landau rule) method.

\section{Numerical Results}

Now, we calculate numerically the contribution of trapped and untrapped electrons to $\operatorname{Im} \epsilon_{\|}^{m, m^{\prime}}$. To have some analogy with the one-mode (cylindrical) approximation of the wave dissipation by electron Landau damping, let us estimate the diagonal $\left(m=m^{\prime}\right)$ elements $\operatorname{Im} \epsilon_{\|, u}^{m, m}$ and $\operatorname{Im} \epsilon_{\|, t}^{m, m}$. The corresponding local cylindrical approximation, $\operatorname{Im} \epsilon_{\|, c}^{m, m}$, has the next form

$$
\operatorname{Im} \epsilon_{\|, c}^{m, m}=\frac{2 \sqrt{\pi} r^{3} \omega_{p o}^{2} \omega}{h_{\theta}^{3}\left|m+n q_{t}\right|^{3} v_{T}^{3}} \exp \left[-\frac{\omega^{2} r^{2}}{h_{\theta}^{2}\left(m+n q_{t}\right)^{2} v_{T}^{2}}\right] .
$$

The estimations of $\operatorname{Im} \epsilon_{\|, u}^{m, m}, \operatorname{Im} \epsilon_{\|, t}^{m, m}$ and $\operatorname{Im} \epsilon_{\|, c}^{m, m}$ are carried out for waves with mode numbers $n=4$ and $m=1$ in a plasma with the main TCABR-parameters: $R=0.61 \mathrm{~m}, a=0.18 \mathrm{~m}, H_{\phi 0}=1 \mathrm{~T}, q_{t}(r)=$ $1.1 /\left(1-0.7 r^{2} / a^{2}\right), N(r)=3 \times 10^{19}\left(1-0.95 r^{2} / a^{2}\right) \mathrm{m}^{-3}$, $T(r)=1000\left(1-0.99 r^{2} / a^{2}\right)$ eV. As shown in Fig. 2, the contributions of untrapped and trapped electrons to $\operatorname{Im} \epsilon_{\|}^{m, m}$ depend substantially on $\epsilon$ and $\omega$. By com- paring $\operatorname{Im} \epsilon_{\|, u}^{m, m}$ and $\operatorname{Im} \epsilon_{\|, t}^{m, m}$, we see that the Landau damping of waves with a high phase velocity, $v_{p h}=$ $\omega r /\left[h_{\theta}\left(m+n q_{t}\right)\right]>v_{T}$ (e.g., lower hybrid waves), is due to the bounce resonant interaction with untrapped electrons. It means that, under the Lower-Hybrid Resonance Heating, the favorable conditions can be created to transform the wave momentum into the momentum of untrapped electrons leading the noninductive current drive. 


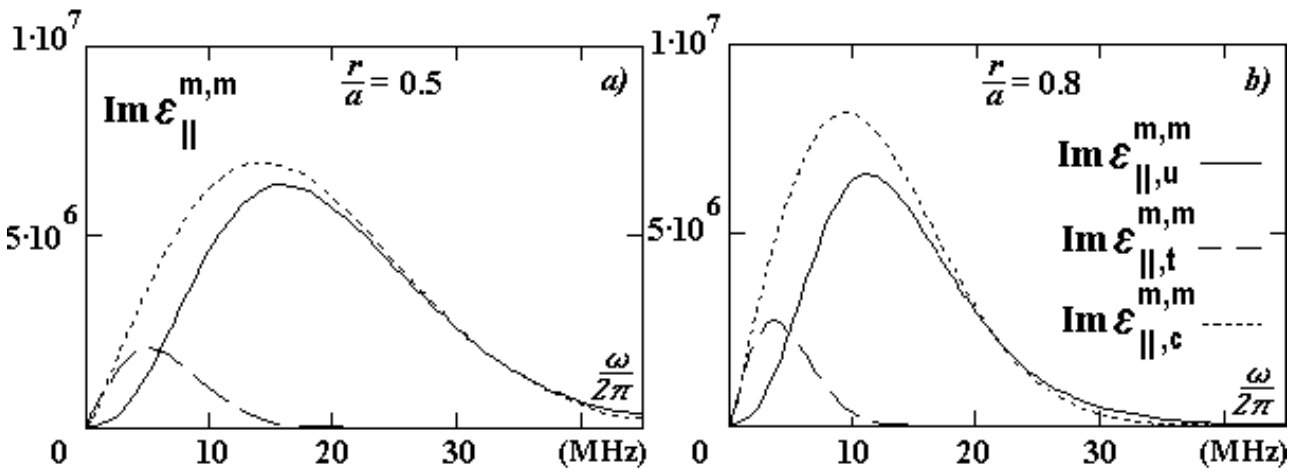

Figure 2. The contribution of untrapped (solid lines), $\operatorname{Im} \epsilon_{\|, u}^{m, m}$, and trapped (long-dashed lines), $\operatorname{Im} \epsilon_{\|, t}^{m, m}$, electrons to the imaginary part of the longitudinal permittivity in a tokamak plasma as a function of wave frequency, $\omega$, for different magnetic surfaces: $a) r / a=0.5, b) r / a=0.8$.

Note, for both the toroidal and cylindrical plasma models, $\operatorname{Im} \epsilon_{\|, u}^{m, m}$ and $\operatorname{Im} \epsilon_{\|, c}^{m, m}$ are exponentially small for the fast waves with $v_{p h}>>v_{T}$. However, the wave dissipation by untrapped electrons can be larger than the corresponding cylindrical approximations at the external magnetic surfaces, where $v_{p h} / v_{T} \sim 2 \div 3$.

Another feature of wave-particle interaction in a toroidal plasma is the role of trapped particles becomes substantial for the slow waves with $v_{p h}<<v_{T}$ under the condition when $\omega$ is comparable with the bounce frequency of the thermal electrons. Moreover, the waves (usually, low-frequency waves) interact effectively with the trapped electrons at the external magnetic surfaces, where the fraction of trapped particles increases as $\sqrt{2 \epsilon /(1+\epsilon)}$. The effective heating of trapped electrons (Fig. 3) is possible in tokamaks using the Alfvén waves, when the local Alfvén resonance condition is realized near the plasma boundary (or in the region of moderate radii).
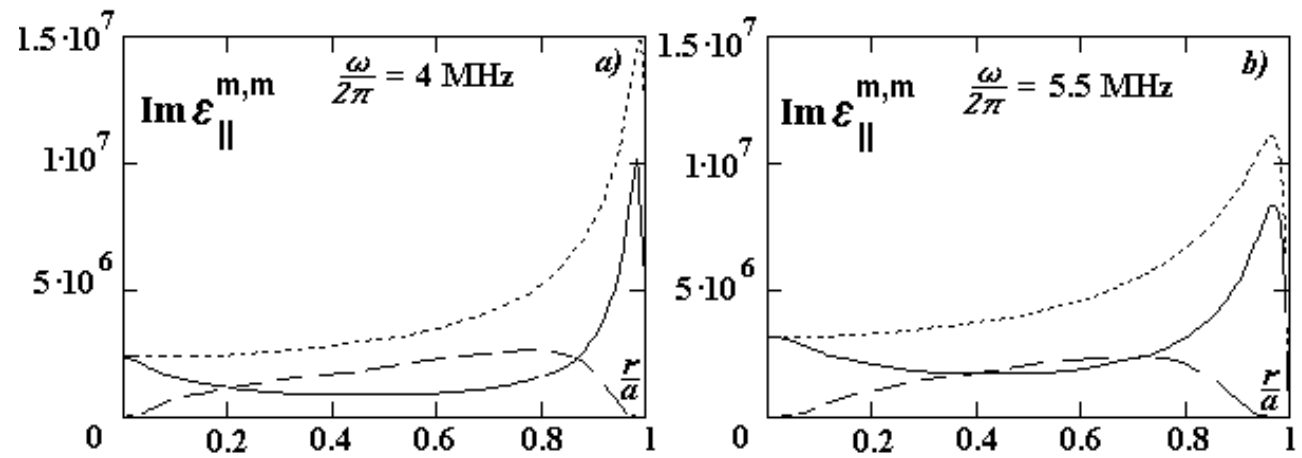

Figure 3. The radial structure of $\operatorname{Im} \epsilon_{\|, u}^{m, m}$ (solid lines), $\operatorname{Im} \epsilon_{\|, t}^{m, m}$ (long-dashed lines), and $\operatorname{Im} \epsilon_{\|, c}^{m, m}$ (short-dashed lines) in the TCABR tokamak plasma for different levels of wave (generator) frequency: $a$ ) $\omega / 2 \pi=4 \mathrm{MHz} ; b) \omega / 2 \pi=5.5 \mathrm{MHz}$.

\section{Conclusion}

The kinetic transverse $(8,9)$ and longitudinal $(10$, 11) permittivity elements have been derived for radiofrequency waves by solving Vlasov equation for trapped and untrapped particles in an axisymmetric toroidal plasma with circular magnetic surfaces and arbitrary aspect ratio. These dielectric tensor components are expressed by summation of bounce-resonant terms, which include the double integration in velocity space, the resonant denominators, the phase coefficients, the standard elementary and elliptic functions. It is shown that the analytical Landau integration (by introducing the plasma dispersion function, or the probability integral of the complex argument) is possible only for the longitudinal permittivity.

Imaginary parts of the longitudinal permittivity elements are important to estimate the power absorbed by electron Landau damping (e.g., during the plasma heating and current drive generation) in the frequency range of Alfvén, fast magnetosonic, and lower hybrid waves. Whereas, imaginary parts of the transverse permittivity elements are necessary to estimate the cyclotron resonant wave dissipation at the fundamental cyclotron frequency of ions and/or electrons. The dissipated wave power is expressed, Eqs. (24) and (27), by summation 
of terms including the separate contributions of trapped and untrapped particles to the imaginary parts of both the diagonal and non-diagonal elements of the dielectric permittivity.

The dielectric tensor components, Eqs. (8-11), can be used for both the Large $(\epsilon<<1)$ and Low $(\epsilon<1)$ Aspect Ratio Tokamaks to analyze the finite- $\epsilon$ effects in the frequency range of Alfvén, Fast Magnetosonic, Lower Hybrid, and Ion/Electron Cyclotron Waves.

\section{Appendix:}

\section{Phase Coefficients by the Jacobi Elliptic Func- tions}

The phase coefficients in Eqs. (8-11) can be calculated by introducing the Jacobi elliptic functions, which are as the standard functions for advanced versions of such mathematical programs as Mathcad, Mathematica, Maple. In particular, for the longitudinal (parallel) dielectric permittivity the coefficients $C_{p}^{m}(\kappa)$ and $D_{p}^{m}(\kappa)$ can be represented as

$$
\begin{array}{r}
C_{p}^{m}(\kappa)=\int_{-K(\kappa)}^{K(\kappa)} \cos \left[2\left(m+n q_{t}\right) \operatorname{am}(\kappa, w)-\left(p+n q_{t}\right) \frac{\pi \hat{\Pi}\left(\kappa_{o}, w\right)}{\hat{\Pi}\left(k_{o}, K(\kappa)\right)}\right] \operatorname{dn}(\kappa, w) d w \\
D_{p}^{m}(\kappa)=\sqrt{\kappa} \int_{-2 K(\kappa)}^{2 K(\kappa)} \cos \left[2\left(m+n q_{t}\right) \arcsin (\sqrt{\kappa} \operatorname{sn}(\kappa, w))-\right. \\
\left.-p \frac{0.5 \pi \hat{\Pi}\left(\kappa_{o} \kappa, w\right)}{\hat{\Pi}\left(\kappa_{o} \kappa, K(\kappa)\right)}\right] \operatorname{cn}(\kappa, w) d w
\end{array}
$$

Here the corresponding Jacobi elliptic functions are

$$
\begin{aligned}
\operatorname{sn}(\kappa, w) & =\frac{2 \pi}{\sqrt{\kappa} K(\kappa)} \sum_{l=0}^{\infty} \frac{\hat{q}^{l+1 / 2}(\kappa)}{1-\hat{q}^{2 l+1}(\kappa)} \sin \frac{(2 l+1) \pi w}{2 K(\kappa)} \\
\operatorname{cn}(\kappa, w) & =\frac{2 \pi}{\sqrt{\kappa} K(\kappa)} \sum_{l=0}^{\infty} \frac{\hat{q}^{l+1 / 2}(\kappa)}{1+\hat{q}^{2 l+1}(\kappa)} \cos \frac{(2 l+1) \pi w}{2 K(\kappa)} \\
\operatorname{dn}(\kappa, w) & =\frac{\pi}{2 K(\kappa)}+\frac{2 \pi}{K(\kappa)} \sum_{l=1}^{\infty} \frac{\hat{q}^{l}(\kappa)}{1+\hat{q}^{2 l}(\kappa)} \cos \frac{l \pi w}{K(\kappa)} \\
\operatorname{am}(\kappa, w) & =\frac{\pi u}{2 K(\kappa)}+\sum_{l=1}^{\infty} \frac{2 \hat{q}^{l}(\kappa)}{l\left(1+\hat{q}^{2 l}(\kappa)\right)} \sin \frac{l \pi w}{K(\kappa)}
\end{aligned}
$$

where the parameter $\hat{q}$ is equal to

$$
\hat{q}(\kappa)=\exp \left[-\pi \frac{K(1-\kappa)}{K(\kappa)}\right],
$$

and the elliptic integral of the third kind in the $(\kappa, w)$ variables has been done as

$$
\hat{\Pi}\left(\kappa_{o}, w\right)=\int_{0}^{w} \frac{d u}{1+\kappa_{o} \mathrm{sn}^{2}(\kappa, u)} .
$$

The phase coefficients $A_{p, l}^{m}(u, \kappa)$ and $B_{p, l}^{m}(u, \kappa)$, for the transverse permittivity elements, may be rewritten by analogy with Eqs. (29) and (30), introducing the new $w$ variable, instead of the angle variable $\theta^{\prime}$ :

$$
w\left(\theta^{\prime}\right)=\int_{0}^{\theta^{\prime} / 2} \frac{d \eta}{\sqrt{1-\kappa \sin ^{2} \eta}}
$$

for untrapped particles, and

$$
w\left(\theta^{\prime}\right)=\int_{0}^{\arcsin \left(\frac{1}{\sqrt{\kappa}} \sin \frac{\theta^{\prime}}{2}\right)} \frac{d \eta}{\sqrt{1-\kappa \sin ^{2} \eta}}
$$

for the trapped particles. 


\section{References}

[1] V.N. Belikov, Ya.I. Kolesnichenko, A.B. Mikhailovskii and V.A. Yavorskii, Sov. J. Plasma Phys. 3, 146 (1977).

[2] F.M. Nekrasov, Sov. J. Plasma Phys. 18, 520 (1992).

[3] F. Porcelli, R. Stancievicz, W. Kerner and H.L. Berk, Phys. Plasmas 1, 470 (1994).

[4] S.V. Kasilov, A.I. Pyatak and K.N. Stepanov, Plasma Phys. Reports 24, 465 (1998).

[5] N.I. Grishanov, C.A. de Azevedo and J.P. Neto, X Int.
Congress on Plasma Phys. \& 42nd Annual APS-DPP Meeting, Quebec, Canada, 2000, Vol. I, p.13 (2001).

[6] N.I. Grishanov, C.A. de Azevedo and J.P. Neto, Plasma Phys. Controlled Fusion 43, 1003 (2001).

[7] F.M. Nekrasov, A.G. Elfimov, C.A. de Azevedo, A.S. de Assis and J.P. Neto, Plasma Phys. Controlled Fusion 43, 727 (2001).

[8] N.I. Grishanov and F.M. Nekrasov, Sov. J. Plasma Phys. 16, 29 (1990). 